\title{
NUMERICAL STUDY ON EFFECTS OF VEGETATION \\ CANOPY IN URBAN AREAS
}

\author{
By PHAM Hong Son ${ }^{*}$ and Takashi ASAEDA *
}

\begin{abstract}
A turbulent closure model including the vegetation effects was developed to simulate the lower atmospheric layer in the urban areas. The submodels based on the SIB (Simple Biosphere Model) were used to calculate the atmospheric parameters at vegetation areas. These submodels used the variables at referent level and the resistances represented the vegetation to calculate new values for next time step and then feedback to the main model. The turbulent model (Yamada) which already being developed to including the effects of the buildings and pavement on the heat transfer process has been used as mainmodel in this study. The observation and numerical study were carried out for Tama Center on the west of Tokyo.
\end{abstract}

Keywords: Heat island, Biosphere model, Urban canopy.

\section{INTRODUCTION}

Urban areas are covered by a large percentage of asphalt and concrete, which are usually dry, water proof surfaces with albedo and heat capacities that convert and store incoming radiation as sensible heat. The cities normally have complicated configuration is composed of high buildings, pavement and greenery areas. The turbulent closure model (Yamada, Mellor 1974) was widely used to study the lower atmospheric layer in the urban areas. In this model the vegetation was considered as drag element in the momentum equations, independently specify the reflectance, drag and energy partition properties, but in nature these properties are closely interconnected by the type of vegetation present; (Sellers et al 1986). The optical properties of the leaves, their size, number and geometric arrangement determine the surface reflectance and surface drag properties, and these together with the vegetation physiology, also determine the vegetation's control on the evapotranspiration rate.

The work of Dickinson (1984) and Seller et al. (1986) has been directed at constructing biosphere models, which aim to model the vegetation itself and thereby calculate consistent reflectance, drag and energy partition characteristics for given vegetated surface. The Simple Biosphere Model (SIB) of Sellers et al. (1986) was designed to describe the climatologically important interactions between the terrestrial biosphere and the atmosphere, specifically, the flux of radiation, momentum, sensible heat and latent (evapotranspiration) heat. The SIB was used to simulate the biosphere processes in many

* Grad. Student, Dept. of Civil and Environmental Sciences, Saitama Univ.

* (255 shimo-okubo, Urawa, Saitama 338).

Associate Prof. Dept. of Civil and Environmental Sciences, Saitama Univ.

(255 shimo-okubo, Urawa, Saitama 338). 
forest areas at macro scale and it's results compare reasonably well with appropriate measurements obtained from the literature. The SIB, however, was very complicate and required more than 50 properties in simulation.

The purpose of this study is to develop a model treats all factors such as vegetation, buildings, pavement and reservoir for use in the urban areas. The SIB was modified to simulate the biosphere properties of vegetation canopy. The Yamada turbulent closure model supplies meteorological variables at referent level and after they are being updated by SIB will feedback to the main model.

\section{MODEL PHILOSOPHY}

A modified version of the turbulent closure model (Yamada) which already developed to including the pavement effects (Asaeda, Vu 1993) has chosen as main model in this study. The energy exchange between the upper climate and the surface covered by asphalt, concrete and concentrated building canopy was simulated to study the impacts of the pavement materials, concentration and height of buildings on the environmental system. The reflection of radiation flux by the buildings and pavement also being considered in the heat exchange process. The green elements in this model were considered only as drag force in the momentum equations. The model structure provided to illustrate the surface configuration for each grid point with pavement, buildings, vegetation, water surface. To simulate the biosphere impacts of the vegetation, the submodels based on SIB (Sellers 1986) was developed for green elements. Since there are some differences in approaching ways between two models, it is favorable to consider separately in simulation. For vegetation covered area, biosphere model controlled from the surface up to the referent height above the tree canopy while the mainmodel controlled remaining volume. The referent layer above tree canopy was boundary for two models. Using the atmospheric properties at referent height provided by the mainmodel, the biosphere submodels calculate the variables in canopy and covered ground.

The differences in calculation the properties between the two models required to modify the biosphere submodels in some aspects. In SIB, the calculation of radiation flux is fairly complex, involving estimation of five components of the incident radiation following the proposal of Dickinson (1983). The variation of albedo also estimated in corresponding with the leaf area index of the vegetation. The vegetation in the urban area normally well arranged and configuration is simpler than the forest area. In this study, the radiation flux already provided by turbulent model and simply used it to calculate the net radiation fluxes on canopy and ground. The tree albedo was referred as constant in corresponding with the field measurement.

The treatment for the two layers of vegetation in SIB complicates the structure scheme in transformation the morphological and physiological characteristics of the vegetation canopy into the model parameters. 'I he green elements in the urban areas are normally less densely arranged and the lower story canopy (shrubs) covered only small percentage of the surface. Therefore, for sake of simplicity, only upper story (high tree) has been treated in present study.

Another simplification has been made in term of deep soil layer. SIB divided the soil into three layers to govern the moisture wetness storage. Herein, only one surface layer was considered and temperature distribution of the soil has been estimated by solving the conduction heat equation knowing the surface soil temperature. 


\section{STRUCTURE OF BIOSPHERE MODEL}

The model was designed to use the meteorological variables from the referent layer to calculate the fluxes of radiation, sensible heat, latent heat and momentum on the vegetated surface. The morphological and physiological characteristics of the vegetation community at a grid point are parameterized and used to derive the coefficients and resistances which govern the fluxes between surface and atmosphere.

The main concept of the model is a description the sensible and latent heat fluxes in the resistant form. In the case of the sensible heat fluxes, the potential differences are $\left(T_{c}-T_{a}\right)$ for the canopy and $\left(T_{s}-T_{a}\right)$ for the ground, where $T$ is the temperature and subscribes $c, s, a$, refer to the canopy, ground and air in the air canopy space respectively.

A given grid point has independently specified the canopy height, leaf area index, moisture storage, covered percentage area. The configuration of the canopy depends on day of the year but for calculating duration, which lasts for about several days, it can be treated independently. Therefore, calculation of the resistor's coefficients using this configuration is carried out only for one time at the beginning of the calculation.

The three aerodynamic resistances; $r_{a}$ : between canopy air space and referent height, $\mathrm{sm}^{-1}$; $r_{b}$ : bulk boundary layer, $\mathrm{sm}^{-1}$ and $r_{d}$ : between the ground and air canopy space, $\mathrm{sm}^{-1}$ conduct correspondence to the turbulent transfer coefficient for heat and vapor. They are derived from calculations involving the morphology of the vegetation and soil surface, the meteorological variables at the referent height. The detailed calculation was described in Sellers (1986). The equations govern the sensible heat fluxes are given by:

From canopy vegetation to canopy air space

$$
H_{c}=\frac{2\left(T_{c}-T_{a}\right)}{r_{b}} \rho c_{p}
$$

From ground to canopy air space

$$
H_{s}=\frac{\left(T_{s}-T_{a}\right)}{r_{d}} \rho c_{p}
$$

From canopy air space to reference height

$$
H_{c}+H_{s}=\frac{\left(T_{a}-T_{r}\right)}{r_{a}} \rho c_{p}
$$

where $r$ is air density $\mathrm{kgm}^{-3}$ and $c_{p}$ is specific heat of air, $\mathrm{Jkg}^{-1} \mathrm{~K}^{-1}$.

For the latent heat fluxes $\lambda E$, all the potential differences can be expressed in terms of the temperatures, $T_{C}$, $T_{S}$ the moisture stores, $M_{C}, M_{S}$, and the atmospheric variables at referent level. $r_{C}$ : bulk stomata resistance of upper story vegetation, $\mathrm{sm}^{-1} ; r_{g}$ : bulk stomata resistance of ground vegetation $\mathrm{sm}^{-1}$; and $r_{\text {surf }}$. bare soil surface resistance $\mathrm{sm}^{-1}$, imposed on the transfer of water vapor from the saturated tissues within the leaves of the canopy and ground cover vegetation and from the water within the soil surface store respectively. The surface resistances are calculated from a combination of plant physiological and morphological parameters, the state of the surface soil moisture store, the atmospheric boundary conditions and the values of the prognostic variables (see Sellers 1986 for detailed description). These resistances are sensitive and are required to compute in each time step. The bulk stomata resistance $r_{c}$ : is defined as

$$
\frac{1}{r_{c}}=V_{c} N_{c} f(\Sigma) \int_{0}^{L_{t}} \frac{G}{r_{s}} d L
$$

in which $G$ is canopy leaf projection (Goudriaan 1977), $r_{S}$ is stomatal resistance of an 
individual leaf following Javis (1976), $V_{C}$ is cover fraction, $N_{C}$ is green leaf fraction, $f(\Sigma)$ is adjustment factor for the influence of leaf water potential, leaf temperature and water vapor pressure deficit and $L_{t}$ is leaf area index. The latent heat fluxes may be written as follows: From canopy vegetation to canopy air space

$\lambda E_{c}=\left[e_{*}\left(T_{c}\right)-e_{a}\right] \frac{\rho c_{p}}{\gamma}\left[\frac{W_{c}}{r_{b}}+\frac{1-W_{c}}{r_{b}+r_{c}}\right]$

From ground to canopy air space

$\lambda E_{s}=\left[e_{*}\left(T_{s}\right)-e_{a}\right] \frac{\rho c_{p}}{\gamma}\left\{V_{g}\left[\frac{W_{g}}{r_{d}}+\frac{1-W_{g}}{r_{d}+r_{g}}\right]+\left[\frac{\left(1-V_{g}\right) h_{s}}{r_{\text {surf }}+r_{d}}\right]\right\}$

From canopy air space to reference height

$\lambda E_{c}+\lambda E_{s}=\frac{\left(e_{a}-e_{r}\right)}{r_{a}} \frac{\rho c_{p}}{\gamma}$

where $e^{\star}\left(T_{C}\right)$ is saturation water vapor pressure at temperature $T_{C}, \mathrm{mb}, e_{a}$ is water vapor pressure of the air at the canopy source height, $m b, e^{*}\left(T_{S}\right)$ is saturation water vapor pressure at temperature $T_{S}, \mathrm{~K}, V_{g}$ is fractional cover of ground vegetation, $W_{g}$ is wetted fraction of ground cover, $h_{S}$ is factor that adjusts for the relative humidity of the air at the soil surface, $e_{r}$ is water vapor pressure of the air at the reference level within the atmospheric boundary layer, $\mathrm{mb}, \gamma$ is psychrometric constant, $\mathrm{mbK}^{-1}$.

The governing equations for energy exchange between canopy, ground and the atmosphere layer are:

$C_{c} \frac{\partial T c}{\partial t}=R n_{c}-H_{c}-\lambda E_{c}$

$C s \frac{\partial T s}{\partial t}=R n s-H s-\lambda E s$,

where $R n_{C}, R n_{S}$ are absorbed net radiation by canopy and ground respectively $\mathrm{Wm}^{-2}$ and are given in the form:

$R n_{c}=R_{c}-2 \sigma T_{c}^{4} V_{c} \delta_{t} \varepsilon_{c}+\sigma T_{s}^{4} V_{c \delta_{t}} \varepsilon_{s}$

$R n_{s}=R_{s}-\sigma T_{s}^{4} \varepsilon_{s}+\sigma T_{c}^{4} V_{c} \delta_{t} \varepsilon_{c}$

in which $R_{C}, R_{S}$ are the absorbed radiation for the canopy and ground respectively, $\sigma$ is Stefan-Boltzmann constant, $\mathrm{Wm}^{-2} \mathrm{~K}^{-4}, \varepsilon_{\mathcal{C}}, \varepsilon_{S}$ are emissivity of canopy and ground respectively, $\delta_{\tau}$ is canopy transmittance for thermal infrared radiation, $V_{c}$ is fractional area covered by the canopy. Equations (7) and (8) are used to estimate temperature of the canopy and surface for each time step and afterward the other variables.

\section{SITE STUDY}

Tama newtown located on the West of Tokyo was selected for the study. A site where measurements were recorded was a $1.5 \times 1.5 \mathrm{~km}^{2}$ included commercial, resident and green areas. The elevation of the site ranged from $90 \mathrm{~m}$ to $120 \mathrm{~m}$. There are a number of high buildings, large paved areas and green elements that influent on the thermal characteristics of the district. The daily air temperature at $1.5 \mathrm{~m}$ height was recorded (July, 24,1994) at a number of selected points. The energy parameters such as radiation flux, albedo, sensible heat also being measured at Center Park. Fig. 1 showed the measured temperature 
distribution at the study area. The results at some degree reflected the district configuration. The recorded temperature had a highest value at pavement area, where the ground was dry and without any canopy. It reduced in the commercial area and the minimum values were recorded at green area.

The turbulent closure model (Mellor, Yamada) which already being developed to including the heat effects of the buildings and pavement (Asaeda, $\mathrm{Vu}$ 1993) was used as a main model. The referent level above the vegetation canopy was boundary between main and biosphere models. For the vegetated grid point, the biosphere submodels use the data at referent height from the main model to find momentum transfer parameters and then the vertical velocity profile from surface to referent level which include velocity in the canopy, under and above the canopy (Seller 1986) and then estimate the new value of the resistors for given time step. These resistors are used to calculate the energy components for canopy and ground. After that, the temperature of the canopy, air canopy and ground could be updated for next time step. The main model used the new temperature values for calculation upper canopy level and temperature of the deep soil levels.

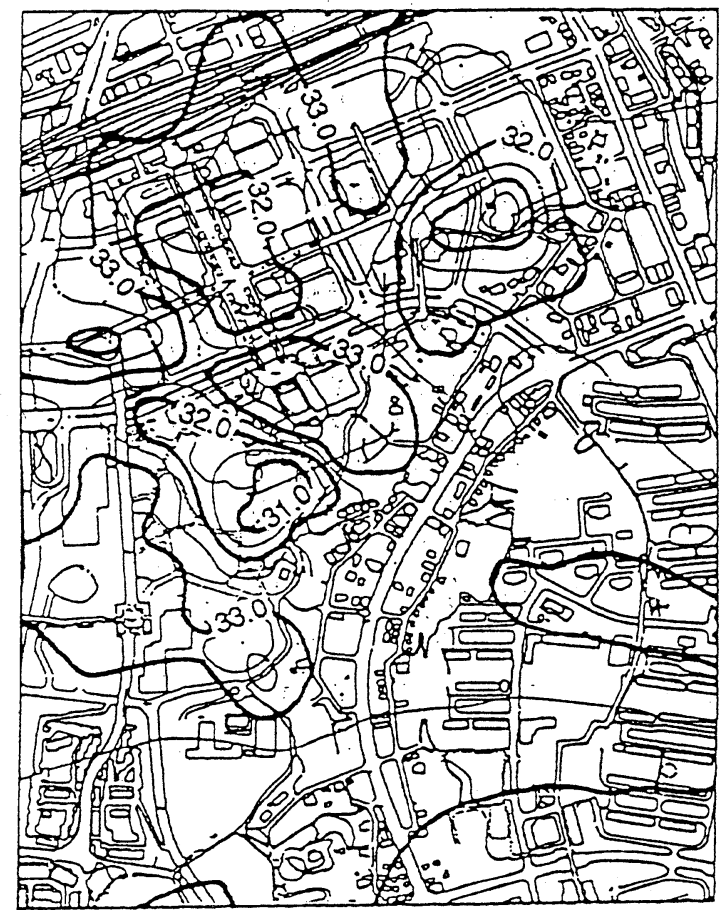

Fig. 1. Measured Temperature at 14:00 h July, 24, 1994

Table 1. Initial condition and parameters set for SIB

\begin{tabular}{|c|c|c|c|}
\hline Definition & Symbol & Units & \\
\hline Initial Condition & & & \\
\hline temperature & $T_{a}$ & ${ }^{\circ} \mathrm{C}$ & 24.0 \\
\hline wind direction & & $\mathrm{S}-\mathrm{N}$ & \\
\hline humidity & & $\%$ & 80 \\
\hline Physiological parameters set for SIB & & & \\
\hline canopy leaf area index & $L_{t}$ & & 5.03 \\
\hline fractional area covered by canopy & $V_{C}$ & $m^{2} m^{-2}$ & 0.8 \\
\hline green leaf fraction & $N_{C}$ & & 0.949 \\
\hline light dependent stomatal & $a$ & $\mathrm{Im}^{-3}$ & 1223.0 \\
\hline response parameters & $b$ & $W_{m}-2$ & 20.9 \\
\hline response parameters & c & $\mathrm{sm}^{-1}$ & 25.2 \\
\hline leaf drag coefficient & $C_{d}$ & & 0.4 \\
\hline leaf shelter factor & $p_{S}$ & & 3.0 \\
\hline canopy heat capacity & $C_{C}$ & $\mathrm{Jm}^{-2} \mathrm{~K}^{-1}$ & $2.0 \times 10^{4}$ \\
\hline ground heat capacity & $C_{S}$ & $\mathrm{Jm}^{-2} \mathrm{~K}^{-1}$ & $2.5 \times 10^{5}$ \\
\hline
\end{tabular}

A calculation was carried out for the $1.5 \times 1.5 \mathrm{~km}^{2}$ with a mesh of $50 \times 50 \mathrm{~m}^{2}$ starting from $6: 00$ on July 24,1994 . The initial condition and SIB parameters are given in the table 1 . 
Surface boundary conditions for wind, temperature and water vapor are constructed trom the empirical formulas by Dyer and Hicks (1970).

For the area covered by vegetation, the biosphere model controls up to the referent level. and the turbulent model governs the space above referent level. For each time step, the velocity at referent height was used to calculate the wind profile from surface up to referent level and then together with aerodynamic coefficients calculate the resistances. After that, the energy fluxes on canopy and ground were derived and by solving energy exchange equation to obtain new value of temperature for next time step before returning to the mainmodel. Fig. 2 showed the calculated temperature distribution at 14:00. The results are at some degree in a good agreement with the recorded data. The thermal distribution mainly depends on the canopy (vegetation, buildings) and surface material (soil, grass, pavement, water). In particular, the results show that the temperature at vegetation cover reduced about 1-2 degree in comparison with sunshine places.

\section{CONCLUSION}

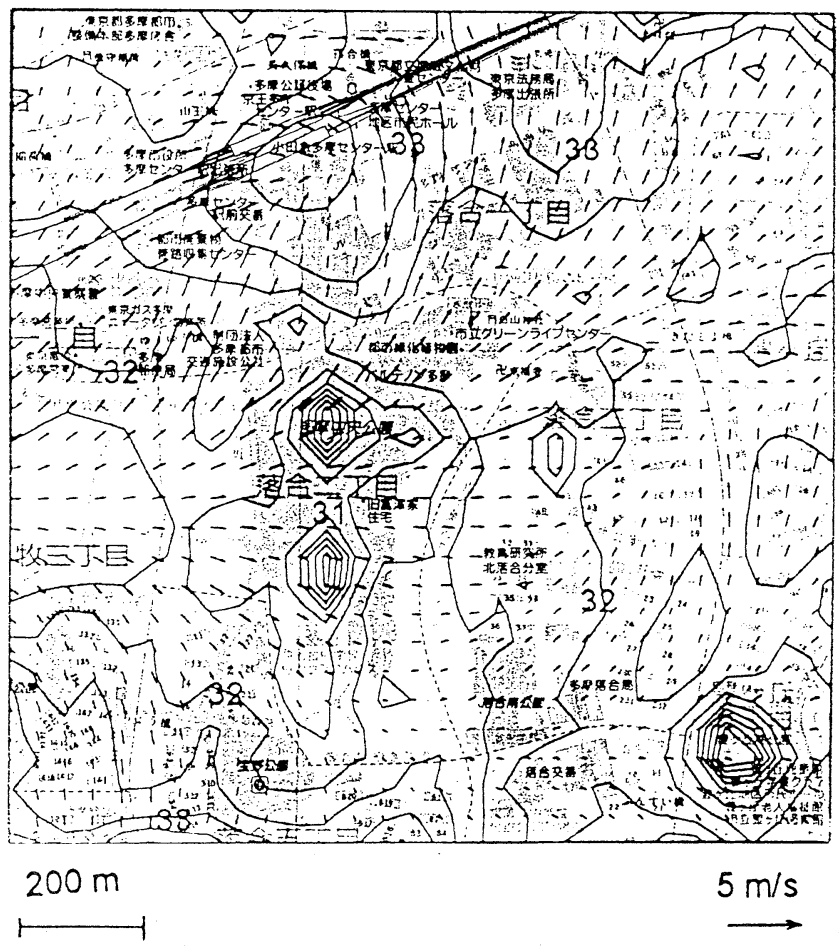

Fig. 2. Calculated Temperature and wind distribution at $14: 00^{\mathrm{h}}$

A numerical model which calculates the air temperature, wind distribution within a district was developed by adding the biosphere activities of the vegetation. The climate characteristics of the district were predicted under a variety of canopy conditions, in particular, the vegetation parameters and their activities were included.

\section{REFERENCES}

1) Dorman, J. L., and Sellers, P. J., 1989: A Global Climatology of Albedo, Roughness Length and Stomatal Resistance for Atmospheric General Circulation Models as Represented by Simple Biosphere Model (SIB), J. Applied Meteorology, 28, 833-855.

2) Sellers, P. J., Mintz, Y., Sud, Y. C., and Dalcher, A., 1986: A Simple Biosphere Model (SIB) for use within General Circulation Models, J. atmospheric Sciences, 43, 505-531.

3) Mellor, G. L. and Yamada, T., 1974: A Hierarchy of Turbulence Closure Models for Planetary Boundary Layer, J. Atmos. Sci., 31, 1791-1806.

4) Garratt, J. R., 1992, The Atmospheric Boundary Layer, Cambridge University Press.

5) Yamada, T., and Bunker, S., 1988, Development of a Nested Grid, Second Moment Turbulence Closure Model and Application to the 1982 ASCOT Brush Creek Data Simulation, J. of Applied Meteorology, Vol. 27. No 5. 562-578.

6) Yamada, T., (1982), A Numerical Model Study of Turbulent Airflow in and above a Forest Canopy, J. of the Meteorological Society of Japan, 60, 439454. 